\title{
The General Exact Bijective Continuous Solution of Feigenbaum's Functional Equation
}

\author{
Patrick J. McCarthy \\ Department of Mathematics, Bedford College, University of London, Regent's Park, \\ London NW1 4NS, England
}

\begin{abstract}
Solutions $f: \mathbb{R} \rightarrow \mathbb{R}$ of Feigenbaum's functional equation $f(f(x))$ $=\alpha^{-1} f(\alpha x)$, where $\alpha \neq 0$ is a fixed real number, account for many of the fascinating properties of the behaviour of successive iterates of (one parameter families of) nonlinear maps. In connection with the phenomenon of intermittency, interesting families of exact solutions have recently been found (for $\alpha>0$ ). These solutions can all be derived from continuous bijective solutions which are topologically equivalent to translations. In this paper, the general exact continuous bijective solution is found for any $\alpha \neq 0$, positive or negative. In particular, it is shown that, for any $\alpha \neq 0$, there are solutions which are inequivalent to translations. And it is shown that bijective solutions equivalent to translations exist only when $0<\alpha<1$. These results considerably enlarge the stock of available exact solutions of Feigenbaum's equation.
\end{abstract}

\section{Introduction}

It is a remarkable fact that, for a wide class of real valued functions $g$ of a real variable, the recursion relations $x_{n+1}=K g\left(x_{n}\right)$ exhibit a rich qualitative [1] and quantitative [2] behaviour which is essentially independent of the recursion function $g$. Feigenbaum [3] and others [4-7] have provided an explanation of the scaling and universal properties of the transition to chaos via period doubling transformations in terms of a functional equation

$$
f_{0}\left(f_{0}(x)\right)=\alpha^{-1} f_{0}(\alpha x)
$$

for a real valued function $f_{0}$ of a real variable, where $\alpha<0$ is a fixed real number. Solutions of $(F)$ are evidently fixed points, in an appropriate function space, of the transformation $T$ defined by

$$
(T f)(x)=\alpha f\left(f\left(\alpha^{-1} x\right)\right),
$$

and the universal properties are [3-7] derived from the behaviour of $T$ near a fixed point in certain eigendirections in function space. 
In the original applications [3-7] of Feigenbaum's equation, the solutions $f$ of (F) of interest were not one-one, but rather, typically, were even functions, and the values of $\alpha$ concerned were negative. However, Hirsch et al., [8] have recently given a formulation of the phenomenon of intermittency in the presence of noise. While the boundary conditions concerned are rather different from Feigenbaum's, their treatment was again based on the behaviour of $T$ near fixed points, but now with $\alpha>0$ (in fact with $\alpha=2$ ). Equation (F) is simpler in this situation. Remarkably, Hirsch et al. [8] found closed form exact solutions of $(F)$ which fitted the boundary conditions, and these used solutions to find the relevant eigenfunctions and eigenvalues.

$\mathrm{Hu}$ and Rudnik [9], in the same context of intermittency, gave further exact solutions of $(\mathrm{F})$, but now with arbitrary positive $\alpha$. Their family of solutions includes those of [8]. For $0<\alpha<1$, the solutions are real analytic and bijective. For $1<\alpha<\infty$, the solutions are again real analytic, but local in nature (being undefined at certain points of $\mathbb{R}$ ) and unbounded. All of the solutions of $[8,9]$ can be derived from continuous, bijective solutions of $(F)$ which are equivalent to translations. In fact, for $\alpha=\frac{1}{2}$, Feigenbaum's equation admits the very simple solutions $f(x)=x+a$, where $a$ is a real constant, and the solutions of $[8,9]$ are all obtained from these simple solutions. The same simple solutions will play a key rôle below.

The purpose of this paper is to give the general continuous exact bijective solutions of Feigenbaum's equation for any $\alpha \neq 0$, positive or negative. This family of solutions includes those of $[8,9]$. A fortioti, the family must contain all real analytic solutions. However, no criteria are given for characterising the entire subfamily of real analytic solutions.

The main tool which will be used below is a criterion for two increasing bijections $g, g^{\prime}$ from $\mathbb{R}$ onto $\mathbb{R}$ to be conjugate, (the conjugator being an increasing bijection). This criterion has long been known (recent references are $[10,11]$ ). It will be shown that bijective solutions of $(\mathrm{F})$ equivalent to translations exist only when $0<\alpha<1$; hence the local nature of the solutions of $[8,9]$ for $1<\alpha<\infty$ is inevitable. Furthermore it will be shown that, for any $\alpha \neq 0$, there are solutions of (F) which are inequivalent to translations.

\section{Exact Solutions of $[8,9]$}

Hirsch et al. [8] gave an exact solution of $(F)$ (the subscript on $f_{0}$ is dropped henceforth) for $\alpha=2$,

$$
f(f(x))=\frac{1}{2} f(2 x)
$$

in the form

$$
f(x)=x(1-a x)^{-1}
$$

where $a$ is any real constant. Hu and Rudnik [9] gave a solution of (F) for $\alpha=2^{y}$ (for any real $y \neq 0$ )

$$
f(f(x))=2^{-y} f\left(2^{y} x\right)
$$


in the form

$$
f(x)=\left(x^{-1 / y}-a\right)^{-y}=x\left(1-a x^{1 / y}\right)^{-y} .
$$

Here the power function $x^{y}$ is defined, for positive, negative or zero $x$, by

$$
x^{y}=\operatorname{sgn}(x)|x|^{y}, \quad(x \neq 0 \text { if } y<0),
$$

where the sign function $\operatorname{sgn}(x)$ is $+1,0$ or -1 for $x>0, x=0$ or $x<0$. Direct substitution shows that (4), where defined, gives an exact solution of (3). And, for the case $y=1$, one gets the solution (2) of (1).

I now rederive the solutions (4) of $\mathrm{Hu}$ and Rudnik [9] (by a method equivalent to theirs), and set up some notation to be used later. Let $T_{a}, M_{\alpha}$, and $P_{\tau}$ denote the (everywhere defined) translation, multiplication and power maps, given as follows

$$
\begin{gathered}
T_{a}(x)=x+a \quad(a \neq 0), \\
M_{\alpha}(x)=\alpha x \quad(\alpha \neq 0 \text { or } 1), \\
P_{\tau}(x)=\operatorname{sgn}(x)|x|^{\tau} \quad(\tau>0, \tau \neq 1) .
\end{gathered}
$$

Observe that the cases in which these maps give the identity map are excluded. Write $T=T_{1}(a=1)$. Denoting function composition by juxtaposition, and inverse maps as usual, evidently $M_{\alpha^{-1}}=M_{\alpha}^{-1}$ and $(\mathrm{F})$ is (for $\alpha>0$ )

$$
f^{2}=M_{\alpha}^{-1} f M_{\alpha}
$$

Now observe that $T=T_{1}$ satisfies

$$
T^{2}=T_{2}=M_{1 / 2}^{-1} T M_{1 / 2},
$$

and so $f=T$ satisfies (F) for $\alpha=\frac{1}{2}$. Next observe that, if $f$ satisfies (F), so does $g=M_{\beta}^{-1} f M_{\beta}$ for any $\beta \neq 0$, since $M_{\beta}$ commutes with $M_{\alpha}$. Hence

$$
M_{\beta}^{-1} T M_{\beta}=T_{1 / \beta}=T_{a} \quad(a=1 / \beta)
$$

also satisfies $(\mathrm{F})$ for $\alpha=\frac{1}{2}$. And an obvious computation shows directly that $T_{a}^{2}=M_{1 / 2}^{-1} T_{a} M_{1 / 2}$.

The power and multiplication maps satisfy the relation

$$
P_{\tau}^{-1} M_{\alpha} P_{\tau}=M_{\alpha^{\prime}}, \quad \alpha^{\prime}=\operatorname{sgn}(\alpha)|\alpha|^{1 / \tau} .
$$

Hence, by conjugating either side of $(\mathrm{F})$ with $P_{\tau}$, one finds that, if $f^{2}=M_{\alpha}^{-1} f M_{\alpha}$, then

$$
g^{2}=M_{\alpha^{\prime}}^{-1} g M_{\alpha^{\prime}}, \quad \alpha^{\prime}=\alpha^{1 / \tau},
$$

where $g=P_{\tau}^{-1} f P_{\tau}$. In particular, the solution $f=T_{a}$ for $\alpha=\frac{1}{2}$ gives rise to a solution $g=P_{\tau}^{-1} T_{a} P_{\tau}$ of $(\mathrm{F})$ for $\alpha=\left(\frac{1}{2}\right)^{1 / \tau}=2^{-1 / \tau}$. That is, by choosing $\tau$ appropriately, the particular solution $f=T_{a}$ for $\alpha=\frac{1}{2}$ can be used to give a solution $g=P_{\tau}^{-1} T_{a} P_{\tau}$ for any $\alpha$ with $0<\alpha<1$.

All of the solutions so far obtained are everywhere defined (bijective continuous) solutions. Further local solutions can be obtained as follows. Let $I$ denote the inverse forming map, defined everywhere except at $x=0$, by

$$
I(x)=x^{-1}, \quad x \neq 0 .
$$


Then one finds that, whenever both sides are defined,

$$
I^{-1} M_{\alpha} I=M_{\alpha^{-1}}, \quad I^{-1} P_{\tau} I=P_{\tau} .
$$

By conjugating either side of (F) with $I$ it follows that, if $f^{2}=M_{\alpha}^{-1} f M_{\alpha}$, then $g^{2}=M_{\alpha^{-1}}^{-1} g M_{\alpha^{-1}}$, where

$$
g=I^{-1} f I .
$$

Hence, from any solution $f$ of $(\mathrm{F})$ with $0<\alpha<1$ one obtains a local solution $g=I^{-1} f I$ with $1<\alpha<\infty$. In particular,

$$
g=I^{-1}\left(P_{\tau}^{-1} T_{a} P_{\tau}\right) I=P_{\tau}^{-1}\left(I^{-1} T_{a} I\right) P_{\tau}
$$

satisfies $(F)$ with $\alpha=\left[2^{-1 / \tau}\right]^{-1}=2^{1 / \tau}$. In detail, this is

$$
g(x)=\left(x^{-\tau}+a\right)^{-1 / \tau} \text {. }
$$

This is the solution (4) of $\mathrm{Hu}$ and Rudnik [9] for $\tau=1 / y$ (with $a$ replaced by $-a$ ). The above derivation shows that the local nature of the solution can be understood as a consequence of the local nature of $I$ in (12).

\section{Preliminaries and the Conjugacy Criterion}

Every continuous bijection $g: \mathbb{R} \rightarrow \mathbb{R}$ is a strictly increasing or strictly decreasing homeomorphism, and every conjugate $h^{-1} g h$ by such a map $h: \mathbb{R} \rightarrow \mathbb{R}$ is increasing (decreasing) whenever $g$ is increasing (respectively, decreasing). It follows that every continuous bijection $f: \mathbb{R} \rightarrow \mathbb{R}$ which solves $(\mathrm{F})$ is increasing. For $f^{2}$ is always increasing, so the conjugate $M_{\alpha}^{-1} f M_{\alpha}$, and hence $f$ itself, must be increasing. The first result deals with trivial solutions.

3.1. Proposition. For any $\alpha \neq 0$, the identity function $f=1$ (defined by $1(x)=x$, all $x \in \mathbb{R}$ ) satisfies $(\mathrm{F})$. For $|\alpha|=1$, every solution of $(\mathrm{F})$ is the identity function $f=1$.

Proof. It is immediate that $f=1$ satisfies (F) for any $\alpha \neq 0$. Suppose next that $\alpha=1$, so that $f(f(x))=f(x)$. Since $f$ is onto, every $y \in \mathbb{R}$ is of the form $y=f(x)$ for some $x \in \mathbb{R}$. But then $f(y)=y$, and $f$ is the identity function. Finally suppose that $\alpha=-1$, so that $(F)$ is $f^{2}=J^{-1} f J$, where $J=M_{-1}$ is defined by $J(x)=-x$. It follows that $f^{4}$ $=J^{-1} f^{2} J=J^{-2} f J^{2}=f$. Every $y \in \mathbb{R}$ is of the form $y=f(x)$ for some $x \in \mathbb{R}$, so $f^{3}(f(x))=f^{3}(y)=f(x)=y$. Suppose that, for some $y \in \mathbb{R}, f(y) \neq y$, say $f(y)>y$. Since $f$ preserves order, this gives $f^{2}(y)>f(y)$ and $f^{3}(y)>f^{2}(y)$. Thus $y=f^{3}(y)>f^{2}(y)$ $>f(y)>y$, a contradiction. Similarly $f(y)<y$ leads to a contradiction. Hence $f(y)$ $=y$ for all $y \in \mathbb{R}$, so $f$ is the identity function. The proof is finished.

Henceforth, only those $\alpha$ will be considered with $|\alpha| \neq 1$. In fact, until Sect. 6 , only positive values of $\alpha$ will be considered. Henceforth, $A(I)$ will denote the group, order function composition, of increasing self homeomorphisms of an open interval $I$ (possibly infinite) of $\mathbb{R}$, and $A$ will often denote $A(\mathbb{R})$. The next result shows that, to solve (F) for any $\alpha>0$, it is enough to solve (F) in the special cases $\alpha=\frac{1}{2}$ and $\alpha=2$. 
3.2. Proposition. (A) Suppose $0<\alpha<1$. Then the general solution $f \in A$ of $f^{2}$ $=M_{\alpha}^{-1} f M_{\alpha}$ is obtained from the general solution $g \in A$ of $g^{2}=M_{1 / 2}^{-1} g M_{1 / 2}$ by the formula $f=P_{\tau} g P_{\tau}^{-1}$, where $\tau>0$ is the unique number with $\alpha^{-1 / \tau}=2$.

(B) Suppose $1<\alpha<\infty$. Then the general solution $f \in A$ of $f^{2}=M_{\alpha}^{-1} f M_{\alpha}$ is obtained from the general solution $g \in A$ of $g^{2}=M_{2}^{-1} \mathrm{gM}_{2}$ by the formula $f=P_{\sigma} g P_{\sigma}^{-1}$, where $\sigma>0$ is the unique number with $\alpha^{1 / \sigma}=2$.

Proof. This follows from formula (10) by conjugating either side of $f^{2}=M_{\alpha}^{-1} f M_{\alpha}$ with the appropriate power function. For $0<\alpha<1, P_{\tau}^{-1} M_{\alpha} P_{\tau}=M_{1 / 2}$, and for $1<\alpha$ $<\infty, P_{\sigma}^{-1} M_{\alpha} P_{\sigma}=M_{2}$. The proof is finished.

Because of the special role of the multiplication operators $M_{1 / 2}$ and $M_{2}$, it will be convenient to write, henceforth, $M=M_{1 / 2}$ and $N=M_{2}$. For any given multiplication operator $M_{\alpha}$, let $\mathscr{M}_{\alpha}$ denote the subgroup of $A=A(\mathbb{R})$ of elements which commute with $M_{\alpha}$;

$$
\mathscr{M}_{\alpha}=\left\{m \in A \mid m M_{\alpha}=M_{\alpha} m\right\} .
$$

In particular, write $\mathscr{M}=\mathscr{M}_{1 / 2}$ and $\mathscr{N}=\mathscr{M}_{2}$. An obvious way to generate new solutions from old is given by

3.3. Proposition. For some given $\alpha \neq 0$, let $f \in A$ be a solution of $f^{2}=M_{\alpha}^{-1} f M_{\alpha}$. Then $f^{\prime}=m^{-1} f m, m \in \mathscr{M}_{\alpha}$, is also a solution of the same equation.

Proof. This follows immediately by conjugating both sides of the equation with $m$, giving $f^{\prime 2}=M_{\alpha}^{-1} f^{\prime} M_{\alpha}$.

It will also be convenient to define, for each translation $T_{a} \in A$, the group

$$
\mathscr{T}_{a}=\left\{t \in A \mid t T_{a}=T_{a} t\right\}
$$

In particular, write $\mathscr{T}=\mathscr{T}_{1}$. Both groups, $\mathscr{M}_{\alpha}$ and $\mathscr{T}$, will be seen to play a rôle in describing the full solution space of $(\mathrm{F})$ in $A=A(\mathbb{R})$.

For a given open interval $I$, the group $A(I)$ may be described as the group of order preserving bijections of $I$. To analyse bijective solutions of $(F)$, I will use a long known conjugacy criterion (recent references: $[10,11]$ ) in $A(I)$. With each $g \in A(I)$ associate a function $\theta: I \rightarrow\{1,-1,0\}$ by defining $\theta(x)=1$ whenever $g(x)>x$, $\theta(x)=0$ whenever $g(x)=x$ and $\theta(x)=-1$ whenever $g(x)<x$. The fixed point set $S \subset I$ of $g$ is the closed set $S=\{x \mid g(x)=x\}$, which is the zero set $\theta^{-1}(0)$ of $\theta$. Often, $\theta$ will be called the indicator function of $g$. Like all open subsets of $\mathbb{R}$, the complement $M=I-S$ decomposes into a countable disjoint union of open intervals $\mu_{j}$, where $j$ ranges over a countable index set. It is easy to see that $g$ restricts to a countable family of component maps $g_{j} \in A\left(\mu_{j}\right)$ with $g_{j}(x)>x$ (all $\left.x \in \mu_{j}\right)$ or $g_{j}(x)<x\left(\right.$ all $\left.x \in \mu_{j}\right)$. That is, these maps are fixed point free homeomorphisms of the intervals $\mu_{j}$, and $\theta$ restricts to a constant map $\theta_{j}$ of $\mu_{j}$ in each (with value 1 or -1 , respectively). The required criterion is given by $[10,11]$.

3.4. Theorem. $g, g^{\prime} \in A(I)$ are conjugate in $A(I)$ if and only if there is an element $k \in A(I)$ such that $\theta k=\theta^{\prime}$.

Proof. Suppose first that $g, g^{\prime}$ are conjugate; $g^{\prime}=k^{-1} g k$ for some $k \in A(I)$. Suppose, in turn, that $\theta^{\prime}(x)=1,-1$ or 0 . So $g^{\prime}(x)>x, g^{\prime}(x)<x$ or $g^{\prime}(x)=x$. That is $\left(k^{-1} g k\right)(x)$ 
$>x,<x$ or $=x$. Hence $(g k)(x)>k(x),-<k(x)$ or $=k(x)$, respectively. Therefore $\theta k=\theta^{\prime}$.

Conversely, suppose that $g, g^{\prime} \in A(I)$ are such that, for some $k \in A(I), \theta k=\theta^{\prime}$. Define $g^{\prime \prime}=k^{-1} g k$ so that, as in the first section, $\theta k=\theta^{\prime \prime}$. Since $g$ and $g^{\prime \prime}$ are conjugate, it is enough to prove that $g^{\prime}$ and $g^{\prime \prime}$ are conjugate, using the fact that $\theta^{\prime}$ $=\theta^{\prime \prime}$. The last equation implies that $g^{\prime}$ and $g^{\prime \prime}$ have the same fixed point set $S$, and $\theta_{j}^{\prime}=\theta_{j}^{\prime \prime}$ in each $\mu_{j}$. Select a particular $\mu=\mu_{j}$, and suppose for definiteness that $\theta_{j}(x)=1$ for $x \in \mu_{j}$. Choose a pair of points $p . q \in \mu_{j}$ and let $K, L$ denote the intervals $\left(p, g_{j}^{\prime}(p)\right],\left(q, g_{j}^{\prime \prime}(q)\right]$. Then, using the fact that $g_{j}^{\prime}, g_{j}^{\prime \prime}$ preseve order, it is easily checked that the intervals $\left(g_{j}^{\prime}\right)^{m}(K), m \in \mathbb{Z}$, are disjoint. And these intervals cover $\mu_{j}$, for $\lim _{m \rightarrow \pm \infty}\left(g_{j}^{\prime}\right)^{m}(p)$ must be the right (left) endpoint of $\mu_{j}$, otherwise $\mu_{j}$ would contain a fixed point of $g^{\prime}$. Similarly, the intervals $\left(g_{j}^{\prime \prime}\right)^{m}(L)$ are disjoint and cover $\mu_{j}$. Let $\alpha: K \rightarrow L$ be the unique (necessarily increasing) affine map from $K$ onto $L$. Each $x \in \mu_{j}$ belongs to $\left(g_{j}^{\prime}\right)^{m}(K)$ for exactly one $m \in \mathbb{Z}$. Define a function $l_{j}: \mu_{j} \rightarrow \mu_{j}$ by $l_{j}(x)$ $=\left(\left(g_{j}^{\prime \prime}\right)^{m} \alpha\left(g_{j}^{\prime}\right)^{-m}\right)(x)$ for $x \in\left(g^{\prime}\right)^{m}(K)$. Then it is easy to check that $l_{j} \in A\left(\mu_{j}\right)$ and $l_{j} g_{j}^{\prime}$ $=g_{j}^{\prime \prime} l_{j}$. A similar construction works for $\theta_{j}(x)=-1$ for $x \in \mu_{j}$. Now define $h: I \rightarrow I$ by $h(x)=x$ for $x \in S, h(x)=l_{j}(x)$ for $x \in \mu_{j}$. Then one can check that $h \in A(I)$, and one gets $h g^{\prime}=g^{\prime \prime} h$. Hence $g^{\prime}=h^{-1} g^{\prime \prime} h$, and $g^{\prime}, g^{\prime \prime}$ are conjugate. The proof is finished.

3.5. Corollary. An important special case of this theorem is given as follows: Every fixed point free map $g \in A$ (i.e. $S$ is empty) with $\theta=1$ in $M=\mathbb{R}$ is conjugate to every other such map. In particular, every such map $g \in A$ is conjugate to $T \in A$, since the translation $T(x)=x+1$ is fixed point free and has $\theta=1$. Thus one has the useful canonical form for such maps;

$$
g=k^{-1} T k, \text { some } k \in A .
$$

Similarly, every fixed point free map $g^{\prime} \in A$ with $\theta=-1$ is conjugate to every other such map, and can be written

$$
g^{\prime}=k^{-1} T^{-1} k, \text { some } k \in A .
$$

3.6. Corollary. Every map $g \in A$ with exactly one fixed point, and with $\theta=1(-1)$ in the interval to the right (respectively, left) of the fixed point is conjugate to every other such map, and has the canonical form (here $N=M_{2}$ )

$$
g=k^{-1} N k \text {, some } k \in A \text {. }
$$

Similarly, every map $g^{\prime} \in A$ with exactly one fixed point, and with $\theta=-1(1)$ in the interval to the right (respectively, left) of the fixed point is conjugate to every other such map, and has the canonical form (here $M=M_{1 / 2}$ )

$$
g^{\prime}=k^{-1} M k, \text { some } k \in A .
$$

3.7. Corollary. Suppose that $m$ is a positive integer, and that the maps $\alpha, \beta \in A$ are related by $\alpha^{m}=\beta$. Then $\alpha$ and $\beta$ are conjugate. Indeed, it is easy to check that the indicator functions of $\alpha$ and $\beta$ must coincide, and the result then follows from Theorem 3.4 (compare [10-12]). 


\section{Fixed Point Free Solutions of (F), $\alpha>0$}

It will be important to deal first with certain properties of elements of the group $\mathscr{T} C$ A of elements commuting with the translation $T$. The required results are contained in the following two propositions.

4.1. Proposition. Every element $\pi \in \mathscr{T}$ is of the form $\pi(x)=x+p(x)$, where $p(x)$ is a continuous periodic function of period 1 such that $x+p(x)$ is one-one. For every $\pi \in \mathscr{T}$, there are fixed real numbers $r$, such that

$$
x+r \leqq \pi(x) \leqq x+s, \text { all } x \in \mathbb{R} .
$$

Proof. By definition, $\pi T=T \pi$, so $\pi(x+1)=\pi(x)+1$. Define $p(x)$ by $\pi(x)=x+p(x)$. Then $p(x+1)=p(x)$. Since $\pi$ is continuous, so is $p$. Because $[0,1]$ is compact, $p(x)$ attains its upper and lower bounds $s$ and $r$ in $[0,1]$, and because $p$ is periodic of period $1, r \leqq p(x) \leqq s$ for all $x \in \mathbb{R}$. The desired inequality then follows because $\pi(x)$ $=x+p(x)$.

4.2. Remark. It is easy to see that there is a plentiful supply of continuous periodic functions $p$ of period 1 such that $\pi(x)=x+p(x)$ is one-one. For example, $p(x)$ could be any differentiable function of period 1 such that $\frac{d p}{d x}(x)>-1$ for all $x \in \mathbb{R}$.

4.3. Proposition. Suppose $\pi \in \mathscr{T}$ and $M=M_{1 / 2}$. Then the function $\pi M$ has the following properties: (A) $\pi M$ has at least one fixed point. (B) There are $\pi$ 's for which $\pi M$ has exactly one fixed point. (C) Whenever $\pi M$ has exactly one fixed point, the indicator function $\theta$ is equal to $-1(+1)$ to the right (left) of the fixed point.

Proof. (A) Since $(\pi M)(x)=\pi\left(\frac{1}{2} x\right)$, (23) gives the inequality $\frac{1}{2} x+r \leqq(\pi M)(x) \leqq \frac{1}{2} x+s$. Hence $(\pi M)(x)-x$ satisfies $-\frac{1}{2} x+r \leqq(\pi M)(x)-x \leqq-\frac{1}{2} x+s$. The extreme left and right of this inequality are both positive (both negative) for sufficiently small (large) $x$, so the same is true of the middle term. Hence, for some $x$, the middle term is zero; $(\pi M)(x)=x$, and the assertion is proved. For $(\mathrm{B})$, observe that examples are given by taking $\pi(x)=x+p(x)$, with $p(x)$ periodic of period 1 , and differentiable, with $-1<\frac{d p}{d x}<1$ for all $x \in \mathbb{R}$. For $(C)$, observe that the indicator must be constant to the right (left) of the fixed point, and $(\pi M)(x)-x$ is negative (positive) for sufficiently large (small) $x$, as remarked above.

The next two theorems deal with fixed point free solutions of $(F)$ for $\alpha>0$.

4.4. Theorem. Suppose $\alpha>1$. Then there are no fixed point free solutions $f \in A$ of $f^{2}$ $=M_{\alpha}^{-1} f M_{\alpha}$.

Proof. By Proposition 3.2(B), no generality is lost by supposing $\alpha=2$. So the equation is $f^{2}=N^{-1} f N$, where $N=M_{2}$. Suppose that there is a fixed point free solution $f$. One may suppose $\theta=1$ for this $f$, for if $\theta=-1, k=f^{-1}$ has $\theta=1$ and satisfies (F) (by inverting both sides of the equation). Since $f$ is fixed point free and has indicator $\theta=1,3.5 \mathrm{implies}$ that, for some $g \in A, f=g \mathrm{Tg}^{-1}$. Substituting into the equation gives

$$
g T^{2} g^{-1}=N^{-1} g T g^{-1} N .
$$


But, by Eq. (9), $T^{2}=M^{-1} T M$, where $M=M_{1 / 2}$. So

$$
\left(g^{-1} N g\right) M^{-1} T=T\left(g^{-1} N g\right) M^{-1} .
$$

That is, for some function $\pi \in A$ with $\pi T=T \pi$ (i.e. $\pi \in \mathscr{T}$ )

$$
g^{-1} N g=\pi M \text {. }
$$

By Corollary 3.6, the left hand side of (24) has exactly one fixed point $p \in \mathbb{R}$, and has indicator function $\theta=1$ to the right of $p, \theta=-1$ to the left of $p$. By Proposition 4.3(B) there are functions $\pi \in \mathscr{T}$ such that $\pi M$ has exactly one fixed point. But, from Proposition $4.3(\mathrm{C}), \pi M$ has indicator function $\theta=-1$ to the right of this point, $\theta=1$ to the left of this point. Hence (24) is impossible. The contradiction establishes the theorem.

4.5. Remark. This result applies to the solutions $(2),(4)$ of $[8,9]$ (for which $\alpha>1$ ). These solutions are not everywhere defined, and Theorem 4.4 shows that this is inevitable if continuous bijective solutions are sought. Many local solutions with $\alpha>1$ (including those of $[8,9]$ ) can be generated from the solutions described in the- next theorem; see Remark 4.8.

By Proposition 3.2(B), to find the general solution $f \in A$ of $(\mathrm{F})$ for $0<\alpha<1$, it is enough to restrict attention to $\alpha=\frac{1}{2}$.

4.6. Theorem. Suppose $\alpha=\frac{1}{2}$, and write $M=M_{1 / 2}$. Then the general fixed point free solution $f \in A$ of $f^{2}=M^{-1} f M$ is

$$
f=g T g^{-1} \text { or } f=g T^{-1} g^{-1},
$$

where $\pi \in \mathscr{T}$ is any element for which $\pi M$ has exactly one fixed point, and $g \in A$ is any element such that $g^{-1} M g=\pi M$.

Proof. If $f$ is fixed point free and satisfies $f^{2}=M^{-1} f M$, one may suppose that $\theta=1$, for otherwise $f^{-1}$ satisfies the same equation and has $\theta=1$. So it is enough to prove that the first formula of (25) gives the general solution with $\theta=1$. By Corollary 3.5, $f=g T g^{-1}$ for some $g \in A$. Substituting into $f^{2}=M^{-1} f M$ gives

$$
g T^{2} g^{-1}=M^{-1} g T g^{-1} M .
$$

But, from Eq. (9), $T^{2}=M^{-1} T M$. Hence

$$
g M^{-1} T M g^{-1}=M^{-1} g T g^{-1} M .
$$

Rearranging gives

$$
\left(g^{-1} M g M^{-1}\right) T=T\left(g^{-1} M g M^{-1}\right) .
$$

That is, for some $\pi \in \mathscr{T}$ (i.e. $\pi T=T \pi$ )

$$
g^{-1} M g=\pi M .
$$

By Corollary 3.6, the left hand side has exactly one fixed point, with $\theta=-1(\theta=1)$ to the right (left) of this point. Hence the same must be true of the right hand side $\pi M$. By Proposition 4.3(B), there are maps $\pi \in \mathscr{T}$ such that $\pi M$ has these properties; so $\pi$ must be such a map. By Proposition $4.3(\mathrm{C})$, the indicator function 
$\theta$ of $\pi M$ for such $\pi$ is $-1(+1)$ to the right (left) of the fixed point. But then, by Corollary 3.6 [Eq. (22)], $\pi M$ is conjugate to $M$ in $A(\mathbb{R})$. That is, Eq. (26) is soluble for $g$; the explicit method of solution is given in Theorem 3.4. Let $g$ be any solution; then

$$
f=g T g^{-1}
$$

is the general solution of $(\mathrm{F})$ with $\theta=1$. The proof is complete.

Recall from Proposition 3.3 that, if $f$ solves $f^{2}=M^{-1} f M$, so does $f^{\prime}=m^{-1} f m$ for any $m \in \mathscr{M}$. Call two solutions which are related in this way $\mathscr{M}$-related. The next result shows that a given $\pi$ gives rise to a family of $\mathscr{M}$-related solutions.

4.7. Theorem. For $\alpha=\frac{1}{2}$, fixed point free solutions of $f^{2}=M^{-1} f M$ are parametrised by spaces of $\mathscr{T}$-cosets of the form $\mathscr{M} g_{\pi} / \mathscr{T}$, where $g_{\pi}$ is a particular solution of (26) for a given admissible $\pi \in \mathscr{T}$.

Proof. Let $g_{\pi}$ be a particular solution of (26), so $g_{\pi}^{-1} M g_{\pi}=\pi M$, and let $h$ be any other solution; $h^{-1} M h=\pi M$. Then $h g_{\pi}^{-1} M g_{\pi} h^{-1}=h \pi M h^{-1}=M$, so $h g_{\pi}^{-1}=m$, where $m M=M m$. That is, the general solution of (26) is $h=m g_{\pi}$ for some $m \in \mathscr{M}$. By (27), two such solutions $h, h^{\prime}$ give rise to the same $f$ if and only if $h^{\prime}=h \tau$ for some $\tau \in \mathscr{T}$. Hence, for a given $\pi \in \mathscr{T}$ such that $\pi M$ has exactly one fixed point, the solutions are parametrised by $\mathscr{M} g_{\pi} / \mathscr{T}$.

4.8. Remark. Particularly simple fixed point free solutions may be found as follows. If $\pi$ is the identity function, an obvious solution of (26) is given by taking $g=M_{\beta}$, the operator of multiplication by $\beta$. Then (27) becomes $f=M_{\beta} T M_{\beta}^{-1}=T_{\beta}$. This solves (F) for $\alpha=\frac{1}{2}$. A local solution $f^{\prime}$ for $\alpha=2$ is therefore, by (14), given by $f^{\prime}$ $=I^{-1} f I=I^{-1} T_{\beta} I$, where $I(x)=x^{-1}(x \neq 0)$. This, when power conjugated as in Proposition 3.2(B), is the Hu-Rudnik solution. Many more local solutions for $1<\alpha<\infty$ are given by $f^{\prime}=I^{-1} f I$, with $f$ defined by (27) (with appropriate power conjugation understood).

It is easy to see that another simple possibility, namely that of taking $\pi(x)$ $=x+k$, where $k$ is a constant (i.e. $\pi$ is the translation map $\pi=T_{k}$ ), merely has the effect of replacing $g$ by $g T_{2 k}$. By (27), this leaves the solution $f$ unchanged.

4.9. Remark. It is easy to see that each map $m \in \mathscr{M}_{\alpha},(\alpha>0)$, determines, and is determined by, a pair of maps $\sigma^{+}, \sigma^{-} \in \mathscr{T}$. Indeed, applying $m M_{\alpha}=M_{\alpha} m$ to the origin $0 \in \mathbb{R}$ gives $m(0)=0$. But $m$ preserves order so restricts to a pair of maps $m^{+} \in A\left(\mathbb{R}^{+}\right), m^{-} \in A\left(\mathbb{R}^{-}\right)$. Let $E_{\alpha}: \mathbb{R} \rightarrow \mathbb{R}^{+}$be the homeomorphism $E_{\alpha}(x)=\alpha^{x}$, with $E_{\alpha}^{-1}(x)=\log _{\alpha}(x)$. Then $E_{\alpha}^{-1} M_{\alpha} E_{\alpha}=T$, and conjugation of $m^{+} M_{\alpha}=M_{\alpha} m^{+}$gives $\sigma^{+} T$ $=T \sigma^{+}$, where $\sigma^{+}=E_{\alpha}^{-1} m^{+} E_{\alpha} \in A(\mathbb{R})$. Hence $\sigma^{+} \in \mathscr{T}$. Similarly using $E_{\alpha}^{\prime}: \mathbb{R} \rightarrow \mathbb{R}^{-}$; $E_{\alpha}^{\prime}(x)=-\alpha^{-x}$, one finds $\sigma^{-} T^{-1}=T^{-1} \sigma^{-}$, whence $\sigma^{-}=E_{\alpha}^{\prime-1} m^{-} E_{\alpha}^{\prime} \in \mathscr{T}$. The result follows.

\section{Solutions of (F) with Fixed Points, and $\alpha>0$}

For any given $\alpha>0$, let $G_{\alpha}$ denote the countable group

$$
G_{\alpha}=\left\{M_{\alpha}^{n} \mid n \in \mathbb{Z}\right\}
$$


generated by the multiplication operator $M_{\alpha}$. Obviously $G_{\alpha}$ has three types of orbit in $\mathbb{R}$, namely (i) the origin $0 \in \mathbb{R}$, (ii) the countable collection of isolated points $G_{\alpha}(p)$ $=\left\{M_{\alpha}^{n}(p) \mid n \in \mathbb{Z}\right\}$ for some positive $p \in \mathbb{R}$, and (iii) the countable collection of isolated points $G_{\alpha}(-q)=\left\{M_{\alpha}^{n}(-q) \mid n \in \mathbb{Z}\right\}$ for some negative $-q \in \mathbb{R}(q>0)$. If $\mathbb{R}^{+}=(0, \infty)$ $\left(\mathbb{R}^{-}=(-\infty, 0)\right)$, evidently $G_{\alpha}(p) \subset \mathbb{R}^{+}$and $G_{\alpha}(-q) \subset \mathbb{R}^{-}$.

Suppose that $f \in A(\mathbb{R})$, and let $S$ denote the fixed point set of $f$;

$$
S=\{x \in \mathbb{R} \mid f(x)=x\} .
$$

By Corollary 3.7, the indicator functions of $f$ and of $f^{2}$ coincide. Hence, in particular, the fixed point sets of $f$ and $f^{2}$ coincide. The next result concerns the nature of $S$ for solutions of $(\mathrm{F})$.

5.1. Proposition. Suppose $f \in A(\mathbb{R})$ satisfies $f^{2}=M_{\alpha}^{-1} f M_{\alpha}$ for $\alpha>0$, and has fixed point set $S$. Then if $S$ is nonempty, $S$ contains the origin $0 \in \mathbb{R}$.

Proof. Suppose $S \neq \emptyset$. To prove the second sentence, it is enough to show that, if $s \in S$, then $M_{\alpha}(s) \in S$ and $M_{\alpha}^{-1}(s) \in S$. The fixed point set of $f^{2}$ is also $S$. Apply $f^{2}$ $=M_{\alpha}^{-1} f M_{\alpha}$ to $M_{\alpha}^{-1}(s)$. This gives

$$
f^{2}\left(M_{\alpha}^{-1}(s)\right)=\left(M_{\alpha}^{-1} f\right)(s)=M_{\alpha}^{-1}(s) .
$$

Hence $M_{\alpha}^{-1}(s)$ is fixed by $f^{2}$, whence $M_{\alpha}^{-1}(s) \in S$. Now apply the equation to $s$. This gives

$$
f^{2}(s)=s=\left(M_{\alpha}^{-1} f M_{\alpha}\right)(s) .
$$

Hence $M_{\alpha}(s)$ is fixed by $f$, whence $M_{\alpha}(s) \in S$ and so the set $S$ is a disjoint union of $G_{\alpha}$ orbits in $\mathbb{R}$.

If $r \in S$, either $r=0$ or $r \in \mathbb{R}^{+}$or $r \in \mathbb{R}^{-}$. If $r=0, S$ contains the origin. If not, suppose $r>0$. Then, by the above paragraph, $S$ contains the sequence $r_{m}=M_{\alpha}^{m}(r)$, $m=1,2,3, \ldots$ and also the sequence $r_{m}^{\prime}=M_{\alpha}^{-m}(r)$. If $0<\alpha<1$, the first sequence tends to $0 \in \mathbb{R}$, and if $1<\alpha<\infty$, the second sequence tends to $0 \in \mathbb{R}$. But $S$ is closed, and so $0 \in S$. Similarly, $r<0$ leads to the same conclusion. Hence in all cases, $0 \in S$, and the assertion is proved.

Since $S$ contains the origin $0 \in \mathbb{R}$ and $f \in A(\mathbb{R})$ preserves order, $f(0)=0$ implies that $f$ restricts to a pair of maps $f^{+} \in A\left(\mathbb{R}^{+}\right)$and $f^{-} \in A\left(\mathbb{R}^{-}\right)$, where $\mathbb{R}^{+}=(0, \infty)$ and $\mathbb{R}^{-}=(-\infty, 0)$. The next result concerns these maps.

5.2. Theorem. Suppose $f \in A(\mathbb{R})$ satisfies $f^{2}=M_{\alpha}^{-1} f M_{\alpha}$ and has fixed point set $S \neq \emptyset$, and let $f^{ \pm} \in A\left(\mathbb{R}^{ \pm}\right)$be the restrictions of $f$ to $\mathbb{R}^{ \pm}$. Then neither $f^{+}$nor $f^{-}$is fixed point free.

Proof. Consider first $f^{+} \in A\left(\mathbb{R}^{+}\right)$and let $l: \mathbb{R}^{+} \rightarrow \mathbb{R}$ denote the map $l(x)=\log _{\alpha}(x)$. Observe that $l M_{\alpha} l^{-1}=T \in A(\mathbb{R})$. Conjugate either side of $\left(f^{+}\right)^{2}=M_{\alpha}^{-1} f^{+} M_{\alpha}$ with $l$, giving

$$
g^{2}=T^{-1} g T
$$

where $g=l f^{+} l^{-1} \in A(\mathbb{R})$. If $f^{+}$is fixed point free, so is $g$, and one may assume without loss of generality that $\theta=1$ for $g$. Hence, by Corollary $3.5, g=k^{-1} T k$ for some $k \in A(\mathbb{R})$. Substituting into (28) gives

$$
k^{-1} T^{2} k=T^{-1} k^{-1} T k T .
$$


But $T^{2}=M^{-1} T M$, and rearranging gives $\left(k T k^{-1} M^{-1}\right) T=T\left(k T k^{-1} M^{-1}\right)$. Hence, for some $\pi \in \mathscr{T}$,

$$
k T k^{-1}=\pi M .
$$

By Corollary 3.5, the left hand side is fixed point free. But by Proposition 4.3(A), the right hand side had at least one fixed point. Hence Eq. (29) is impossible, so $f^{+}$ cannot be fixed point free. By a similar argument, $f^{-}$cannot be fixed point free either. The theorem is proved.

To summarise, every solution $f \in A(\mathbb{R})$ of $(\mathrm{F})$ with $s \neq \emptyset$ satisfies $f(0)=0$, and is determined by a pair $f^{+} \in A\left(\mathbb{R}^{+}\right), f^{-} \in A\left(\mathbb{R}^{-}\right)$of solutions of $(\mathrm{F})$, each of which has fixed points. The next result relates $f^{+}, f^{-}$to solutions of (28).

5.3. Proposition. Suppose $f^{+} \in A\left(\mathbb{R}^{+}\right)\left(f^{-} \in A\left(\mathbb{R}^{-}\right)\right)$satisfies $(\mathrm{F})$ and has fixed point $p \in \mathbb{R}^{+}$(respectively, $q \in \mathbb{R}^{-}$). Let $k_{p}: \mathbb{R}^{+} \rightarrow \mathbb{R}$ (respectively, $k_{q}: \mathbb{R}^{-} \rightarrow \mathbb{R}$ ) be the homeomorphism $k_{p}(s)=\log _{\alpha}\left(\frac{x}{p}\right)\left(\right.$ respectively, $\left.k_{q}(x)=\log _{\alpha}\left(\frac{x}{q}\right)\right)$. Then $f^{+}=k_{p}^{-1} g k_{p}$ (respectively, $f^{-}=k_{q}^{-1} g k_{q}$ ) where $g \in A(\mathbb{R})$

$$
g^{2}=T^{-1} g T
$$

and $g$ leaves every point of $\mathbb{Z} \subset \mathbb{R}$ fixed.

Proof. For definiteness, consider $f^{+}$first. Define $g \in A(\mathbb{R})$ by $g=k_{p} f^{+} k_{p}^{-1}$. Conjugating either side of $\left(f^{+}\right)^{2}=M_{\alpha}^{-1} f^{+} M_{\alpha}$ gives $g^{2}=T^{-1} g T$ since $k_{p} M_{\alpha} k_{p}^{-1}=T$. Applying $g k_{p}=k_{p} f^{+}$to $p$ gives $g(0)=0$. The same reasoning as in Proposition 5.1 shows that $f^{+}$leaves $M_{\alpha}^{n}(p)$ fixed for any $n \in \mathbb{Z}$. Hence $g$ leaves $T^{n}(0)$ fixed for any $n \in \mathbb{Z}$. That is, $g$ leaves each point of $n=T^{n}(0)$ of $\mathbb{Z} \subset \mathbb{R}$ fixed. Applying the same reasoning to $f^{-} \in A\left(\mathbb{R}^{-}\right)$, the proposition is proved.

Hence, to find the general solution of (F) with $S \neq \emptyset$, it is enough to solve (30) for functions $g \in A(\mathbb{R})$ leaving $\mathbb{Z}$ pointwise fixed. $f^{+}$and $f^{-}$are then defined by the formulae in Proposition 5.3, and $f(0)=0$. As a preliminary to solving (30), we need the following result.

5.4. Theorem. Given $\beta \in A(I)$, let $A^{\beta}(I)$ denote the centraliser $\{l \in A(I) \mid l \beta=\beta l\}$ of $\beta$. Let $m$ be any positive integer. Then there exists exactly one conjugacy class of $A^{\beta}$ of functions $f \in A^{\beta}(I)$ such that

$$
f^{m}=\beta
$$

Proof. By Corollary 3.7, $\beta^{m}$ is conjugate to $\beta$, so $\beta^{m}=k^{-1} \beta k$ for some $k \in A(I)$. Define $\varphi=k \beta k^{-1}$. Then $\varphi^{m}=k \beta^{m} k^{-1}=\beta$, so $f=\varphi$ solves (P). Also by Corollary 3.7, every solution $f$ of $(\mathrm{P})$ is conjugate to $\beta$, and it follows directly from $(\mathrm{P})$ that every solution $f$ commutes with $\beta$. (It is proved in $[10,11]$ ) that $A(I)$ is divisible. Solutions of $(\mathrm{P})$ are treated very explicitly, and in a wider context, in [12].) Any solution $f$ of $(\mathrm{P})$ will be called an $m^{\text {th }}$ root $\beta^{m^{-1}}$ of $\beta$.

To give the general solution of (30) leaving $\mathbb{Z} \subset \mathbb{R}$ fixed, all that is needed is an application of Theorem 5.4 to the interval $I=(0,1)$. 
5.5. Theorem. Let $n \in \mathbb{Z}$, and define $I_{n}=(n, n+1), I=I_{0}$. Let $\beta \in A(I)$ be any function. Define $g \in A(\mathbb{R})$ by

$$
g(n)=n,\left.\quad g\right|_{I_{n}}=T^{n} \beta^{\left(2^{n}\right)} T^{-n} .
$$

Then $g$ satisfies (30) and leaves $\mathbb{Z}$ pointwise fixed. Every solution of (30) leaving $\mathbb{Z}$ pointwise fixed is obtained in this way.

Proof. The function $g \in A(\mathbb{R})$ given in $(31)$ leaves $\mathbb{Z}$ pointwise fixed by definition, and a simple verification shows that it satisfies (30). Conversely, given any solution $g$ of (30) leaving $\mathbb{Z}$ pointwise fixed, define $\beta=\left.g\right|_{I}$. Then repeated application of (30) and induction show that $g$ is given by (31).

It is interesting to note that, for positive $n$, the arbitrary function $\beta \in A(I)$ uniquely determines the restriction of $g$ to $I_{n}$, but for negative $n, n=-m(m>0)$, the $2^{m}$-th root $\beta^{2-m}$ can be any function from a whole conjugacy class of $A^{\beta}(I)$.

This completes the discussion of solutions of $(\mathrm{F})$ with fixed points. The trivial solutions $f^{+}=1$ or $f^{-}=1$ arise by taking $\beta=1$ in (31). The general solution is obtained from 5.1-5.5.

\section{Solutions of (F) with $\alpha<0$}

If $f \in A(\mathbb{R})$ is fixed point free, it has indicator either $\theta=1(f(x)>x$ for all $x \in \mathbb{R})$ or $\theta=-1(f(x)<x$ for all $x \in \mathbb{R})$. It follows easily for $\alpha<0$, that $M_{\alpha}^{-1} f M_{\alpha}$ has indicator function opposite to that of $f$. But $f^{2}$ has the same indicator function as $f$. Hence, for $\alpha<0$, no fixed point free $f \in A(\mathbb{R})$ can satisfy $(\mathrm{F})$. This leads to

6.1. Proposition. Suppose $\alpha<0$ and $f \in A(\mathbb{R})$ satisfies $f^{2}=M_{\alpha}^{-1} f M_{\alpha}$. Then $f(0)=0$ and $f$ is determined by its restrictions $f^{+} \in A\left(\mathbb{R}^{+}\right), f^{-} \in A\left(\mathbb{R}^{-}\right)$, which satisfy

$$
\begin{aligned}
& \left(f^{+}\right)^{2}=M_{\alpha}^{-1} f^{-} M_{\alpha}, \\
& \left(f^{-}\right)^{2}=M_{\alpha}^{-1} f^{+} M_{\alpha} .
\end{aligned}
$$

Either of $f^{+}, f^{-}$determines the other via these equations, and each satisfies the equation (for $g \in A\left(\mathbb{R}^{+}\right)$or $A\left(\mathbb{R}^{-}\right)$)

$$
g^{4}=M_{\alpha^{2}}^{-1} g M_{\alpha^{2}} .
$$

Proof. By the remarks above, $f$ cannot be fixed point free. By an argument similar to the one in Proposition 5.1, it follows that $f(0)=0$. Hence $f$ restricts to a pair of maps $f^{+} \in A\left(\mathbb{R}^{+}\right), f^{-} \in A\left(\mathbb{R}^{-}\right)$. Applying $f^{2}=M_{\alpha}^{-1} f M_{\alpha}$ to positive $x$ gives (32), and to negative $x$ gives (33). Squaring (32), gives, using (33),

$$
\left(f^{+}\right)^{4}=M_{\alpha}^{-1}\left(f^{-}\right)^{2} M_{\alpha}=M_{\alpha^{2}}^{-1} f^{+} M_{\alpha^{2}},
$$

that is, Eq. (34). Similarly, $f^{-}$satisfies (34). If $f^{+}$is defined to be any solution of (35) and $f^{-}$is then determined by (32);

$$
f^{-}=M_{\alpha}\left(f^{+}\right)^{2} M_{\alpha}^{-1},
$$

it follows that

$$
\begin{aligned}
\left(f^{-}\right)^{4} & =M_{\alpha}\left(f^{+}\right)^{8} M_{\alpha}^{-1}=M_{\alpha} \cdot M_{\alpha^{2}}^{-1}\left(f^{+}\right)^{2} M_{\alpha^{2}} M_{\alpha}^{-1} \\
& =M_{\alpha^{2}}^{-1}\left(M_{\alpha}\left(f^{+}\right)^{2} M_{\alpha}^{-1}\right) M_{\alpha^{2}}=M_{\alpha^{2}}^{-1} f^{-} M_{\alpha^{2}} .
\end{aligned}
$$


Similarly, the rôles of $f^{+}, f^{-}$can be reversed.

Hence it is enough to solve (34) for $g \in A\left(\mathbb{R}^{+}\right)$. A simple modification of the proof of Theorem 5.2 (in which $M$ is replaced by $M_{1 / 4}$, and $T^{4}=M_{1 / 4}^{-1} T M_{1 / 4}$ is used) shows that every solution $g$ of (34) must have fixed points. If $p \in \mathbb{R}^{+}$is such a point, define $h \in A(\mathbb{R})$ by $h=k_{p} g k_{p}^{-1}$, where $k_{p}(x)=\log _{\alpha^{2}}\left(\frac{x}{p}\right)$. Then (34) is equivalent
to

$$
h^{4}=T^{-1} h T
$$

where $h \in A(\mathbb{R})$ leaves $\mathbb{Z} \subset \mathbb{R}$ pointwise fixed. The general solution of (37) is obtained in a manner similar to Theorem 5.5 , and is given by

6.2. Proposition. Let $n \in \mathbb{Z}$, and define $I_{n}=(n, n+1), I=I_{0}$. Let $\beta \in A(I)$ be any function. Define $h \in A(\mathbb{R})$ by

$$
h(n)=n,\left.\quad h\right|_{I_{n}}=T^{n} \beta^{\left(4^{n}\right)} T^{-n} .
$$

Then $h$ satisfies (37) and leaves $\mathbb{Z} \subset \mathbb{R}$ pointwise fixed. Every such solution of (37) is obtained in this way.

Proof. This is an obvious parallel to the proof of Theorem 5.5.

In summary, (38) gives the general solution of (37), which gives the general solution of (34) $\left(f^{+}\right)$, which then defines $f^{-}$via (32). The general exact bijective continuous solution $f$ [necessarily in $A(\mathbb{R})]$ has thus been found for any $\alpha \neq 0$.

\section{References}

1. Metropolis, N., Stein, M.L., Stein, P.R. : J. Comb. Theor. 15, 25-44 (1973)

2. Feigenbaum, M.: J. Stat. Phys. 19, 25-52 (1978)

3. Feigenbaum, M.: J. Stat. Phys. 21, 669-706 (1979)

4. Collet, P., Eckmann, J.-P.: Iterated maps on the interval as dynamical systems. Boston: Birkhäuser 1980

5. Collet, P., Eckmann, J.-P., Lanford, III, O.E. : Commun. Math. Phys. 76, 211-254 (1980)

6. Lanford, III, O.E. : Remarks on the accumulation of period doubling bifurcations. In: Mathmatical problems in theoretical physics. Proceedings, Lausanne 1979. Berlin, Heidelberg, New York: Springer 1980

7. Campanino, M., Epstein, H.: Commun. Math. Phys. 79, 261-302 (1981)

8. Hirsch, J.E., Nauenberg, M., Scalapino, D.J.: Phys. Lett. 87 A, 391-393 (1982)

9. Hu, B., Rudnik, J.: Phys. Rev. Lett. 48, 1645-1648 (1982)

10. Holland, W.C.: Mich. Math. J. 10, 399-408 (1963)

11. Glass, A.M.W.: Ordered permutation groups. In: L.M.S. Lecture Note Series, Vol. 55. Cambridge: Cambridge University Press 1981

12. McCarthy, P.J., Stephenson, W.: Bedford College preprint (1983)

Communicated by O. E. Lanford

Received June 17. 1983: in revised form July 18, 1983

Note added in proof. (1) In the present context, solutions of Feigenbaum's equation are very far from unique; the above results show that they are parametrised by arbitrary functions.

(2) One can also study the functional equations $f^{\lambda}=M_{\alpha}^{-1} f M_{\alpha}$ for any value of $\lambda$ : even nonintegral ones make sense in the context of functions $f \in A(\mathbb{R})$. Feigenbaum's equation is the case $\lambda=2$, and Eq. (34) the case $\lambda=4$. Again $f=T_{a}$ gives a solution for a special value of $\alpha$, namely $\alpha=\lambda^{-1}$. One can find the general solution of $f^{\lambda}=M_{\alpha}^{-1} f M_{\alpha}$ using the above methods. 
